\title{
Existence of quasi-periodic solutions for elliptic equations on a cylindrical domain
}

\author{
Claudia Valls*
}

\begin{abstract}
The elliptic equation $\partial_{t t} u=-\partial_{x x} u-\alpha u-g(u), \alpha>0$ is ill-posed and "most" initial conditions lead to no solutions. Nevertheless, we show that for almost every $\alpha$ there exist smooth solutions which are quasi-periodic. These solutions are anti-symmetric in space, and hence they are not traveling waves. Our approach uses the existence of an invariant center manifold, and the solutions are obtained from a KAM-type theorem for the restriction of the equation to that manifold.
\end{abstract}

Mathematics Subject Classification (2000). 35J60, 35R25, 37C55.

Keywords. Center manifolds, elliptic equations, quasi-periodic solutions.

\section{Introduction}

Elliptic problems in cylindrical domains occur in many situations, such as in the study of the deformation of beams or of inviscid channel flows. These problems lead to elliptic equations in which the axial variable formally takes the role of time. This often allows one to use ideas and techniques from the theory of dynamical systems, and in turn to reduce the study of these systems to that of certain (perhaps multivalued) evolution operators associated to the equation. Although elliptic problems in cylindrical domains may be ill-posed, they may still possesses plenty smooth solutions. Here we consider a particular relevant case of such a system and use ideas and techniques from the theory of dynamical systems to establish the existence of quasi-periodic solutions (which are not periodic). These solutions will be obtained inside a certain invariant center manifold for the dynamics.

We note that it is natural to look for recurrent dynamics inside an invariant submanifold which is not stable nor unstable (since the central behavior does not forbid a priori a recurrence of more local nature). The recurrence corresponds here to quasiperiodic behavior on tori, although of course recurrent behavior may also occur in

\footnotetext{
*Supported by Center for Mathematical Analysis, Geometry, and Dynamical Systems, and through Fundação para a Ciência e a Tecnologia by the Programs POCTI/FEDER, POSI and POCI 2010/Fundo Social Europeu, and the grant SFRH/BPD/14404/2003.
} 
other situations, such as for example in the presence of homoclinic behavior with transverse intersections of stable and unstable manifolds. We note that the study of the existence of quasi-periodic solutions for nonlinear partial differential equations was started somewhat recently by Kuksin [7] and Wayne [12].

In the present context, the approach of reducing the dynamics to a center manifold can be traced back to the work of Kirchgässner [6], where he constructed an invariant center manifold for a semilinear elliptic equation on a strip. The method is now sometimes called Kirchgässner reduction, although of course the applicability of this approach strongly depends on the desired results and appropriate additional techniques may need to be developed in each particular case, particularly due to the infinite-dimensional nature of the problems. For later developments and applications in the context of elliptic problems on cylindrical domains we refer the reader to the works [1], [3], [4], [5], [8], [9], [10] and to the references therein.

More concretely, we work with the equation

$$
\frac{\partial^{2}}{\partial t^{2}} u(t, x)=-\frac{\partial^{2}}{\partial x^{2}} u(t, x)-\alpha u(t, x)+g(u(t, x)), \quad \alpha>0,
$$

where $g$ is any odd real-analytic function of the form

$$
g(u)=b u^{3}+\mathrm{O}\left(u^{5}\right), \quad b \neq 0 .
$$

The equation (1) is an ill-posed modification of the sine-Gordon equation, in the sense that "most" initial conditions do not lead to a solution. Our goal is to show that there exist smooth quasi-periodic solutions (in time) of equation (1) for almost every $\alpha$. Furthermore, we shall see that these solutions are never traveling waves (since they are anti-symmetric in space).

We consider the system (1) on the finite $x$-interval $[0,1]$, with the periodic boundary condition $u(t, 0)=u(t, 1)$ and $(\partial u / \partial t)(t, 0)=(\partial u / \partial t)(t, 1)$ for $0 \leq t<\infty$ and zero mean, i.e., $\int_{0}^{1} u(t, x) d x=0$ and $\int_{0}^{1}(\partial u / \partial t)(t, x) d x=0$. Furthermore, we rewrite it as a Hamiltonian system. Introducing the variables $w=(q, p)$, with $q(t, x)=u(t, x)$ and $p(t, x)=(\partial u / \partial t)(t, x)$, and the Hamiltonian

$$
H(w)=\frac{1}{2} \int_{0}^{1}\left(p^{2}+\alpha q^{2}-(\partial q / \partial x)^{2}\right) d x-\int_{0}^{1} g(q) d x,
$$

the equation (1) can be written in the form

$$
\frac{\partial}{\partial t} w=J \nabla_{w} H(w) \quad \text { with } J=\left(\begin{array}{cc}
0 & 1 \\
-1 & 0
\end{array}\right) .
$$

We first briefly consider the linear variational equation of (1), that is,

$$
\frac{\partial^{2}}{\partial t^{2}} u(t, x)=-\frac{\partial^{2}}{\partial x^{2}} u(t, x)+\alpha u(t, x) .
$$


The functions $\phi_{k}(x)=\cos (2 \pi k x)$ are the basic modes of (3) and the numbers

$$
\mu_{k}(\alpha)= \pm \sqrt{4 \pi^{2} k^{2}-\alpha}
$$

are the corresponding frequencies. Each solution of (3) is a superposition of the basic modes

$$
u(t, x)=\sum_{k \geq 0} \phi_{k}(x)\left\{a_{k} e^{\mu_{k}(\alpha) t}+b_{k} e^{-\mu_{k}(\alpha) t}\right\},
$$

in which $a_{k}$ and $b_{k}$ are determined by the initial data $u_{0}(x)$ and $u_{1}(x)$. We denote by $[\cdot]$ the integer part of a real number and set

$$
w_{k}(\alpha)=\left|\alpha-4 \pi^{2} k^{2}\right|^{1 / 2}, \quad k(\alpha):=\left[\frac{\sqrt{\alpha}}{2 \pi}\right], \quad \Lambda(\alpha):=\{k: 1 \leq k \leq k(\alpha)\} .
$$

We define the full Lebesgue measure set

$$
\mathcal{M}=\{\alpha>0: \sqrt{\alpha} /(2 \pi) \text { is not an integer }\} .
$$

We shall only work with values of $\alpha$ in $\mathcal{M}$ (so that $w_{k}(\alpha) \neq 0$ in order to be able to invert a certain operator later on).

One of the most remarkable characteristics of the linear equation (3) is that there is a finite number of coefficients for which $\mu_{k}(\alpha)$ is purely imaginary: the values of $k$ such that $k \in \Lambda(\alpha)$ and on those values $\mu_{k}(\alpha)= \pm i w_{k}(\alpha)$. These correspond to elliptic behavior on a torus of dimension $k(\alpha)$. The tori come in Cantor families forming finite-dimensional Cantor manifolds through the stationary solution $u \equiv 0$. The invariant manifolds may not persist in the nonlinear system (1) due to the resonances among the nodes and to the strong effect of the nonlinear perturbation for large amplitudes. However, using KAM-type arguments it is possible to establish the persistence, in a sufficiently small neighborhood of the origin, of a Cantor submanifold of tori and that it is only slightly deformed. We point out that this Cantor submanifold covers a measure close to full.

Now we apply the center manifold theory to construct a finite-dimensional manifold invariant under (1). Namely, using work of Mielke in [9, Chapter 2], we can prove for each $r \geq 1$ the existence of a $C^{r}$ center manifold around the origin, $W_{c}$, for the equation (1). For a precise statement of our center manifold theorem and a verification of the conditions which allow us to apply Mielke's results see Appendix B. We list here some important properties of the center manifold:

(1) The space $T_{0} W_{c}$ is the span of the eigenvectors $\phi_{k}(\alpha)_{k \leq k(\alpha)}$, with $k(\alpha)$ finite (see (5)). Thus, the restriction of the dynamics to the center manifold is finitedimensional. We note that the center manifold is precisely where one can expect to have invariant tori persisting. Since in our case the dynamics restricted to the center manifold is finite-dimensional this will allow us to use (finitedimensional) KAM techniques to establish the persistence. 
(2) The dynamics on the center manifold is Hamiltonian with a Hamiltonian that coincides with the restriction of the original one in (2) to the center manifold (see [9] for details).

We now consider a certain modification of the action-angle variables $\left(\hat{I}_{j}, \hat{\theta}_{j}\right)$ for equation (3) (see (14) for the definition) and let $\tau>1$. For each real number $m>0$ we define the set

$$
D_{m}=\left\{\hat{I}_{j}>0: m \leq \hat{I}_{j} \leq 2 m, j \in \Lambda(\alpha)\right\} \times \mathbb{T}^{k(\alpha)} .
$$

We also consider the Diophantine condition

$$
|\langle w(\alpha), k\rangle| \geq \gamma\|k\|^{-\tau} \quad \text { for all } k \in \mathbb{Z}^{k(\alpha)} \backslash\{0\},
$$

where $w(\alpha)=\left(w_{1}^{2}(\alpha), \ldots, w_{k(\alpha)}^{2}(\alpha)\right)$.

We now state our main theorem.

Theorem 1. Given $\tau>1$ and $\gamma>0$, for almost every $\alpha$ such that $w(\alpha)$ satisfies (7), and every $m=m(\alpha)>0$ sufficiently small, all invariant tori with frequencies $w(\alpha)$ persist in the perturbed Hamiltonian (2) restricted to the center manifold, in the sense that there is a map $\phi: \mathbb{T}^{k(\alpha)} \rightarrow D_{m}$ of class $C^{\kappa}$ with $\kappa<l-2 \tau-1$, $l \geq 4 \tau+10$, such that the flow on $D_{m}$ is the image by $\phi$ of the linear flow defined by $w(\alpha)$ on $\mathbb{T}^{k(\alpha)}$.

Incidentally, we note that by a well-known theorem in the book by Siegel and Moser [11, section 16], since $\mu_{k} / \mu_{k_{0}} \notin \mathbb{Z}$ for $1 \leq k \neq k_{0} \leq k(\alpha)$ (see (4)) for every $\alpha \in \mathcal{M}$ with the exception of a countable set, there exists a one-parameter analytic family of periodic orbits in (1) (and thus uncountably many) for almost every $\alpha>0$.

To prove Theorem 1 we shall proceed as follows. First, we obtain a Hamiltonian in infinitely many coordinates $\left\{x_{k}, y_{k}\right\}_{k \geq 1}$, which is real analytic near the origin. The linear equation gives rise to the quadratic Hamiltonian $H_{2}$. The first term in the nonlinearity, $b u^{3}(t, x)$, gives rise to terms of order four, $H_{4}$, while $\mathrm{O}\left(u^{5}(t, x)\right)$ gives rise to terms of order greater or equal to six. Thus,

$$
H=H_{2}+H_{4}+\mathrm{O}\left(\|(x, y)\|^{6}\right), \quad H_{4}=\mathrm{O}\left(\|(x, y)\|^{4}\right),
$$

corresponding to an elliptic fixed point in infinitely many degrees of freedom. Since we are going to use KAM techniques which always require some nondegeneracy conditions, we need to compute the Birkhoff normal form up to order four for (2). The upshot is that, for almost every $\alpha>0$, there exists a change of coordinates so that

$$
H=H_{2}+\bar{H}_{4}+\mathrm{O}\left(\|(x, y)\|^{6}\right), \quad \text { with } \bar{H}_{4}=\sum_{(k, l) \in \Lambda(\alpha) \times \Lambda(\alpha)} \bar{H}_{4, k, l} x_{k} y_{k} x_{l} y_{l} .
$$


Thus, the Hamiltonian is integrable up to order 4. Now, KAM theory comes into play. It is straightforward to verify the nondegeneracy conditions for the KAM theory for almost every $\alpha>0$, without any further assumption (see Appendix A). Then we shall show that, given the nondegeneracy conditions, we can reduce the Hamiltonian in the center manifold to a Birkhoff normal form up to terms of sufficiently high order. By taking $m$ sufficiently small in the definition of the domain $D_{m}$, the Hamiltonian will be a small perturbation of the Birkhoff normal form. This will allow us to reduce Theorem 1 to a suitable quantitative KAM theorem of Zehnder in [13].

It is important to note that the size of the perturbations to which the KAM theorem is applied corresponds to the size of the difference between the Hamiltonian and its Birkhoff normal form. Hence, it is possible to choose the neighborhood in the center manifold, or in $D_{m}$, to be so close to the origin so that all necessary smallness conditions are verified. On the other hand, since the reduction to the Birkhoff normal form involves a change to polar coordinates, which are degenerate near the origin, it is necessary to obtain a quantitative KAM theorem (see Theorem 4 below), which will be used to overcome some problems arising from the polar coordinates.

The paper is organized as follows. In Section 2 the Hamiltonian of (1) is written in infinitely many coordinates. In Section 3, the restriction of the Hamiltonian to the center manifold is transformed into its Birkhoff normal form of order four. In Section 4, we consider the existence of invariant Cantor manifolds for Hamiltonians in this normal form. This finally allows us to prove Theorem 1. The details in the verification of the nondegeneracy conditions are given in Appendix A and a sketch of proof of the theorem concerning the existence of the center manifold is given in Appendix B.

\section{The Hamiltonian}

In this section we verify that equation (1) can be rewritten in Hamiltonian form in terms of the Fourier modes. We shall work with (1) taking only into account the first term of the nonlinearity $g(u(t, x))$, that is, $b u^{3}(t, x)$. The point is that the higher order terms do not contribute to the fourth order Birkhoff normal form. Furthermore, all the computations in this paper concerning the normal form can be done in a formal way because:

(1) the derivatives of the transformed Hamiltonian of certain degree, only depend on the derivatives of the Hamiltonian of lower degrees;

(2) to compute a term in the expansion of the center manifold, we only need to work with a finite number of Fourier modes and thus, it is an algebraic expression of the coefficients of the Hamiltonian. 
These two observations together with the existence of a center manifold, $W_{c}$, guarantee that we can proceed formally ignoring questions of domains of operators and convergence of series.

Let us consider the Hamiltonian for equation (1). We shall work with the variables

$$
q(t, x)=u(t, x) \quad \text { and } \quad p(t, x)=\frac{\partial}{\partial t} u(t, x)
$$

decomposed in Fourier series with respect to the $x$-variable. We recall that we are interested in solutions which are not traveling waves. In order to force that our solutions (the existence of which is claimed in Theorem 1) are not traveling waves we require that $q(t, x)$ is odd in $x$. This leads to restrict our attention to variables of the form

$$
q(t, x)=\sum_{k \geq 1} \lambda_{k}(t) \sin 2 \pi k x, \quad p(t, x)=\sum_{k \geq 1} \beta_{k}(t) \sin 2 \pi k x,
$$

where $\beta_{k}(t)=\lambda_{k}^{\prime}(t)$. The variables $q(t, x)$ and $p(t, x)$ correspond to an invariant subspace of (formal) solutions. We note that the fact that we restrict our attention to this subspace also guarantees that the Birkhoff normal form considered below is not resonant.

By Parseval's identity, the Hamiltonian takes the form:

$$
\begin{aligned}
H= & \frac{1}{2} \int_{0}^{1}\left(p^{2}+\alpha q^{2}-\left(\frac{\partial q}{\partial x}\right)^{2}-g(q)\right) d x \\
= & \frac{1}{2} \sum_{(k, l) \in \mathbb{N}^{2}}\left(\beta_{k} \beta_{l}+\alpha \lambda_{k} \lambda_{l}\right) \int_{0}^{1} \sin (2 \pi k x) \sin (2 \pi l x) d x \\
& -2 \pi^{2} \sum_{(k, l) \in \mathbb{N}^{2}} k l \lambda_{k} \lambda_{l} \int_{0}^{1} \cos (2 \pi k x) \cos (2 \pi l x) d x \\
& -\frac{b}{4} \sum_{(k, l, n, m) \in \mathbb{N}^{4}} \lambda_{k} \lambda_{l} \lambda_{n} \lambda_{m} \int_{0}^{1} \sin (2 \pi k x) \sin (2 \pi l x) \sin (2 \pi n x) \sin (2 \pi m x) d x \\
& -\int_{0}^{1}\left(g(q)-\frac{b}{4} q^{4}\right) d x=H_{2}+H_{4}+R_{1},
\end{aligned}
$$

where

$$
H_{2}=\frac{1}{4} \sum_{k \geq 1}\left(\beta_{k}^{2}-\left(4 \pi^{2} k^{2}-\alpha\right) \lambda_{k}^{2}\right), \quad R_{1}=-\int_{0}^{1}\left(g(q)-\frac{b}{4} q^{4}\right) d x,
$$

that is, $R_{1}$ comprises the terms of order greater or equal 5 and, introducing the notation

$$
k_{\sigma, \rho}^{l, n}=k+(-1)^{\sigma} l+(-1)^{\rho} n, \quad \sigma, \rho \in\{0,1\},
$$


we obtain

$$
H_{4}=\frac{b}{32} \sum_{\substack{(\sigma, \rho) \in\{0,1\}^{2},(k, l, n) \in \mathbb{N}^{3}}}(-1)^{\rho+\sigma} \lambda_{k} \lambda_{l} \lambda_{n} \lambda_{k_{\sigma, \rho}^{l, n}},
$$

where we have used that the Fourier series is real for any $n \geq 1$, and the relation $\lambda_{-n}=-\lambda_{n}$.

Note that the quadratic part $H_{2}$ is positive definite for all $k \in \Lambda(\alpha)$ (see (5)) and negative definite for all other $k$. Moreover, the real Jordan form $J$, in the coordinates $\left\{\lambda_{k}, \beta_{k}\right\}_{k \geq 1}$, is

$$
J=\left(\begin{array}{ccccc}
J_{1} & & & & \\
& J_{2} & & & \\
& & \ddots & & \\
& & & J_{k} & \\
& & & & \ddots
\end{array}\right), \quad J_{k}=\left(\begin{array}{cc}
0 & 1 \\
-1 & 0
\end{array}\right), k \geq 1,
$$

and the symplectic two-form is $\Omega=\sum_{k \geq 1} d \lambda_{k} \wedge d \beta_{k}$.

We are going to make a symplectic change of variables to put the Hamiltonian $\mathrm{H}_{2}$ in diagonal form. To this end we introduce, for $k \geq 1$, the notation

$$
\tau_{k}(\alpha)=i^{\frac{\nu_{k}}{2}}\left|\alpha-4 \pi^{2} k^{2}\right|^{1 / 4}, \quad v_{k}= \begin{cases}0, & \text { if } k \in \Lambda(\alpha), \\ 1, & \text { otherwise }\end{cases}
$$

in which $\Lambda(\alpha)$ is the set of values introduced in (5). Then it is immediate to see that the change of variables $\hat{\Phi}=\left(\Phi_{k}\right)_{\{k \geq 1\}}$ with, for any $k \geq 1$,

$$
\Phi_{k}\left(\lambda_{k}, \beta_{k}\right)=\left(x_{k}, y_{k}\right)=\frac{1}{\sqrt{2}}\left(\frac{\beta_{k}}{\tau_{k}(\alpha)}-i \tau_{k}(\alpha) \lambda_{k}, \tau_{k}(\alpha) \lambda_{k}-i \frac{\beta_{k}}{\tau_{k}(\alpha)}\right)
$$

is a symplectic change of variables with new symplectic two-form

$$
\Omega_{1}=\sum_{k \geq 1} d y_{k} \wedge d x_{k}
$$

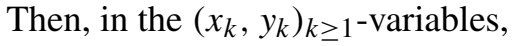

$$
H_{2}=\frac{1}{2} \sum_{k \geq 1} i \tau_{k}^{2}(\alpha) x_{k} y_{k}
$$

and

$$
\begin{aligned}
H_{4}=\frac{b}{128} \sum_{\substack{(\sigma, \rho) \in\{0,1\}^{2} \\
(k, l, n) \in \mathbb{N}^{3}}}(-1)^{\rho+\sigma} \frac{i x_{k_{\sigma, \rho}^{l, n}}+y_{k_{\sigma, \rho}^{l, n}}}{\tau_{k}(\alpha) \tau_{l}(\alpha) \tau_{n}(\alpha) \tau_{k_{\sigma, \rho}^{l, n}}(\alpha)}\left[-i x_{k} x_{l} x_{n}+i y_{k} y_{l} x_{n}\right. \\
\left.\quad-x_{k} y_{l} x_{n}-y_{k} x_{l} x_{n}-x_{k} x_{l} y_{n}+y_{k} y_{l} y_{n}+i x_{k} y_{l} y_{n}+i y_{k} x_{l} y_{n}\right],
\end{aligned}
$$


in which we use the notation introduced in (8).

As it will be clear in Section 3, it is very useful to rewrite $H_{4}$ in another way, different from (11). To this end we set $m=\left(m_{1}, m_{2}, m_{3}, m_{4}\right)$ and $p=\left(p_{1}, p_{2}, p_{3}, p_{4}\right)$, and define the set

$$
A_{4}=\left\{m \in\{0,1\}^{4}, p \in\{0,1\}^{4}: \sum_{i=1}^{4}\left\{m_{i}+p_{i}\right\}=4\right\} .
$$

Then we can write $H_{4}$ in the form

$$
H_{4}=\sum_{\substack{(\sigma, \rho) \in\{0,1\}^{2} \\\left(k, l, n \in \mathbb{N}^{3}, j=k_{\sigma, \rho}^{l, n} \\ m, p \in A_{4}\right.}} h_{k, l, n, j}^{m, p, \sigma, \rho} x_{k}^{m_{k}} x_{l}^{m_{l}} x_{n}^{m_{n}} x_{j}^{m_{j}} y_{k}^{p_{k}} y_{l}^{p_{l}} y_{n}^{p_{n}} y_{j}^{p_{j}}
$$

with

$$
h_{k, l, n, j}^{m, p, \sigma, \rho}=\frac{b(-1)^{\sigma+\rho}}{128 \tau_{k}(\alpha) \tau_{l}(\alpha) \tau_{n}(\alpha) \tau_{j}(\alpha)} \hat{h}_{k, l, n, j}^{m, p},
$$

in which $\hat{h}_{k, l, n, j}^{m, p}$ is equal to either of the following possibilities:

- 1 , if $m_{k}=m_{l}=m_{n}=m_{j}=1$ or $p_{k}=p_{l}=p_{n}=p_{j}=1$.

- -1 , if $p_{k}=p_{l}=m_{n}=m_{j}=1$ or $m_{k}=p_{l}=p_{n}=m_{j}=1$ or $p_{k}=m_{l}=$ $p_{n}=m_{j}=1$ or $m_{k}=p_{l}=m_{n}=p_{j}=1$ or $p_{k}=m_{l}=m_{n}=p_{j}=1$ or $m_{k}=m_{l}=p_{n}=p_{j}=1$.

- $-i$, if $m_{k}=p_{l}=m_{n}=m_{j}=1$ or $p_{k}=m_{l}=m_{n}=m_{j}=1$ or $m_{k}=m_{l}=$ $p_{n}=m_{j}=1$ or $m_{k}=m_{l}=m_{n}=p_{j}=1$.

- $i$, if $p_{k}=p_{l}=p_{n}=m_{j}=1$ or $p_{k}=p_{l}=m_{n}=p_{j}=1$ or $m_{k}=p_{l}=p_{n}=$ $p_{j}=1$ or $p_{k}=m_{l}=p_{n}=p_{j}=1$.

\section{Normal form}

In this section we compute the normal form up to fourth order of the reduction to the center manifold for the Hamiltonian $H=H_{2}+H_{4}+R_{1}$ (introduced in (10) and (11)). Since $R_{1}$ comprises the terms with degree greater or equal to five, only the part of the Hamiltonian given by $\tilde{H}=H_{2}+H_{4}$ will be put in normal form.

Let us introduce, for $\alpha \in \mathcal{M}$ (see (6)),

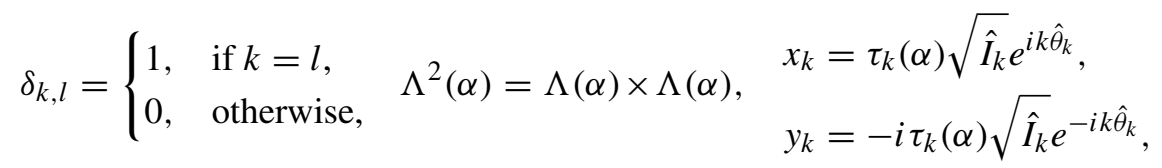

and denote by $\Pi_{W_{c}}(\cdot)$ the projection onto the center manifold. 
Lemma 2. For almost every $\alpha>0$, there exists a symplectic change of coordinates $\Gamma$ that takes the reduction of $H$ to the center manifold into its normal form up to the fourth order. That is

$$
\Pi_{W_{c}}(H)=\bar{H}_{2}+\bar{H}_{4}+R,
$$

where $R$ contains the terms of degree at least five and

$$
\bar{H}_{2}=\frac{1}{2} \sum_{k \in \Lambda(\alpha)}\left|\alpha-4 \pi^{2} k^{2}\right| \hat{I}_{k}, \quad \bar{H}_{4}=-\frac{3 b}{64} \sum_{(k, l) \in \Lambda^{2}(\alpha)}\left(4-\delta_{k, l}\right) \hat{I}_{k} \hat{I}_{l} .
$$

Proof. The proof of Lemma 2 will be done by removing $H_{4}$. According to the remarks in the beginning of Section 2, the corresponding change of variables will be done in a formal way.

Let $\Gamma_{1}=\left.X_{G_{4}}^{t}\right|_{t=1}$ be the time 1-map of the flow of the Hamiltonian vector field $X_{G_{4}}$ given by the Hamiltonian $G_{4}$ defined as:

$$
G_{4}=\sum_{\substack{(\sigma, \rho) \in\{0,1\}^{2} \\(k, l, n) \in \mathbb{N}^{3}, j=k_{\sigma, \rho}^{l, n} \\ m, p \in A^{4}}} g_{k, l, n, j}^{m, p, \sigma, \rho} x_{k}^{m_{k}} x_{l}^{m_{l}} x_{n}^{m_{n}} x_{j}^{m_{j}} y_{k}^{p_{k}} y_{l}^{p_{l}} y_{n}^{p_{n}} y_{j}^{p_{j}}
$$

in which, with the notation,

$$
\mathscr{D}_{k, l, n, j}(\alpha)=\sum_{j=1}^{4} \tau_{\xi_{j}}^{2}(\alpha)\left(m_{\xi_{j}}-p_{\xi_{j}}\right), \quad\left(\xi_{1}, \xi_{2}, \xi_{3}, \xi_{4}\right)=(k, l, n, j),
$$

we have

$$
g_{k, l, n, j}^{m, p, \sigma, \rho}= \begin{cases}0, & \text { if } \mathcal{D}_{k, l, n, j}(\alpha)=0, \\ \frac{2 i h_{k, l, n, j}^{m, p, \sigma, \rho}}{\mathcal{D}_{k, l, n, j}(\alpha)}, & \text { otherwise. }\end{cases}
$$

At this point, in order to explicitly obtain $G_{4}$, we need to compute in which cases $\mathscr{D}_{k, l, n, j}(\alpha)=0$. We can distinguish between the following two situations:

(1) If $m_{\xi_{j}}=p_{\xi_{j}}$, for all $j=1,2,3,4$ with $\left(\xi_{1}, \xi_{2}, \xi_{3}, \xi_{4}\right)=(k, l, n, j)$. In that case, $\mathscr{D}_{k, l, n, j}(\alpha)=0$ for all $\alpha>0$ and $g_{k, l, n, j}^{m, p, \sigma}$ is taken to be equal to zero (see (16)). Moreover, we shall denote by $\mathcal{R}$ the set of values of $(\sigma, \rho, k, l, n, j) \in$ $\{0,1\}^{2} \times \mathbb{N}^{4}$ such that, $j=k_{\sigma, \rho}^{l, n}$ and $m_{k}=p_{k}, m_{l}=p_{l}, m_{n}=p_{n}, m_{j}=p_{j}$ in equation (12).

(2) If $\mathscr{D}_{k, l, n, j}(\alpha)=0$ and condition (1) does not hold. Then we define, for $\alpha \geq 4 \pi^{2}$ (that is, $k(\alpha) \geq 1$ ), the quantity

$$
\omega(\alpha)=\left(\tau_{1}^{2}(\alpha), \ldots, \tau_{k(\alpha)}^{2}(\alpha)\right) .
$$


Notice that $\omega(\alpha)$ is an analytic curve not a straight line in $\mathbb{R}^{k(\alpha)}$ and given $k \in \mathbb{Z}^{k(\alpha)}$, equation (15) vanishes only at locally finite set of points. Let us denote by $\hat{\jmath}$ the complement of this finite set of points. Then $\hat{\jmath}$ has full Lebesgue measure and therefore, in that case, for almost every $\alpha>0, D_{k, l, n, j}(\alpha) \neq 0$.

From now on, we shall work only with the full Lebesgue measure set of values of $\alpha>0$ for which $\mathscr{D}_{k, l, n, j}(\alpha) \neq 0$ in case (2) and, thus, $g_{k, l, n, j}^{m, p, \rho}=0$ if and only if case (1) is satisfied.

It is well known that the transformed Hamiltonian, $\bar{H}$, by the time 1-map of the flow of the Hamiltonian vector field $X_{G_{4}}$ is given by

$$
\begin{aligned}
\bar{H}=H \circ X_{G_{4} \mid t=1}^{t}=H_{2} & +H_{4}+\left\{H_{2}, G_{4}\right\}+R_{1}+\left\{H_{4}+R_{1}, G_{4}\right\} \\
& +\int_{0}^{1}(1-t)\left\{\left\{H, G_{4}\right\}, G_{4}\right\} \circ X_{G_{4}}^{t} d t,
\end{aligned}
$$

in which $\left\{H, G_{4}\right\}$ denotes the Poisson bracket of $H=H_{2}+H_{4}+R_{1}$ and $G_{4}$. Due to the form of $\Omega_{1}$ introduced in (9), the Poisson bracket of $H$ and $G_{4}$ is given by

$$
\left\{H, G_{4}\right\}=\sum_{k \geq 1}\left[\frac{\partial H}{\partial y_{k}} \frac{\partial G_{4}}{\partial x_{k}}-\frac{\partial H}{\partial x_{k}} \frac{\partial G_{4}}{\partial y_{k}}\right] .
$$

It is clear that the last line in (17) comprises terms of degree greater or equal five, and thus the higher order terms. Therefore,

$$
R=\Pi_{W_{c}}\left(R_{1}+\left\{H_{4}+R_{1}, G_{4}\right\}+\int_{0}^{1}(1-t)\left\{\left\{H, G_{4}\right\}, G_{4}\right\} \circ X_{G_{4}}^{t} d t\right) .
$$

Furthermore, by (16),

$$
\Pi_{W_{c}}\left(H_{2}+H_{4}+\left\{H_{2}, G_{4}\right\}\right)=\bar{H}_{2}+\Pi_{W_{c}}\left(\hat{H}_{4}\right),
$$

where

$$
\bar{H}_{2}=\Pi_{W_{c}}\left(H_{2}\right)=\frac{1}{2} \sum_{k \in \Lambda(\alpha)} i \tau_{k}(\alpha)^{2} x_{k} y_{k},
$$

and $\hat{H}_{4}$ is equal to

$$
\begin{aligned}
& \sum_{\substack{(\sigma, \rho) \in\{0,1\}^{2} \\
(k, l, n) \in \mathbb{N}^{3}, j=k_{\sigma, \rho}^{l, n} \\
m, p \in A^{4}}} h_{k, l, n, j}^{m, p, \sigma, \rho} x_{k}^{m_{k}} x_{l}^{m_{l}} x_{n}^{m_{n}} x_{j}^{m_{j}} y_{k}^{p_{k}} y_{l}^{p_{l}} y_{n}^{p_{n}} y_{j}^{p_{j}} \\
& \quad+\sum_{\substack{(\sigma, \rho, k, l, n, j) \notin \mathcal{R}\\
}} \frac{i D_{k, l, n, j}(\alpha) g_{k, l, n, j}^{m, p, \sigma, \rho}}{2} x_{k}^{m_{k}} x_{l}^{m_{l}} x_{n}^{m_{n}} x_{j}^{m_{j}} y_{k}^{p_{k}} y_{l}^{p_{l}} y_{n}^{p_{n}} y_{j}^{p_{j}} \\
& =\sum_{(\sigma, \rho, k, l, n, j) \in \mathcal{R}} h_{k, l, n, j}^{m, p, \sigma, \rho} x_{k}^{m_{k}} x_{l}^{m_{l}} x_{n}^{m_{n}} x_{j}^{m_{j}} y_{k}^{p_{k}} y_{l}^{p_{l}} y_{n}^{p_{n}} y_{j}^{p_{j}} .
\end{aligned}
$$


In order to be able to compute (20), we need to obtain the set $\mathcal{R}$. A necessary (not sufficient) condition is that $\sum_{j=1}^{4} m_{j}=\sum_{j=1}^{4} p_{j}$. This condition together with equation (13) imply that $\hat{H}_{4}$ at least reduces to

$$
\begin{array}{r}
\hat{H}_{4}=-\frac{b}{128} \sum_{\substack{(\sigma, \rho) \in\{0,1\}^{2} \\
(k, l, n) \in \mathbb{N}^{3}, j=k_{\sigma, \rho}^{l, n}}} \frac{(-1)^{\sigma+\rho}}{\tau_{k}(\alpha) \tau_{l}(\alpha) \tau_{n}(\alpha) \tau_{j}(\alpha)}\left[y_{k} y_{l} x_{n} x_{j}+x_{k} y_{l} y_{n} x_{j}\right. \\
\left.+y_{k} x_{l} y_{n} x_{j}+x_{k} y_{l} x_{n} y_{j}+y_{k} x_{l} x_{n} y_{j}+x_{k} x_{l} y_{n} y_{j}\right] .
\end{array}
$$

Furthermore, not all the values of $(\sigma, \rho) \in\{0,1\}^{2},(k, l, n) \in \mathbb{N}^{3}$ and $j=k_{\sigma, \rho}^{l, n}$ in (21) belong to $\mathcal{R}$. To specify more the domain $\mathcal{R}$, we shall proceed as follows:

(1) If $\sigma=0$ and $\rho=0$. This case does not provide any term in $\mathcal{R}$.

(2) If $\sigma=0$ and $\rho=1$. In this case we can rewrite (21) in the following way:

$$
\begin{array}{r}
\hat{H}_{4}=\frac{b}{128} \sum_{(k, l, n) \in \mathbb{N}^{3}} \frac{1}{\tau_{k}(\alpha) \tau_{l}(\alpha) \tau_{n}(\alpha)}\left\{y _ { k } y _ { l } x _ { n } \left(\frac{x_{k+l-n}}{\tau_{k+l-n}(\alpha)}+\frac{x_{n+l-k}}{\tau_{n+l-k}(\alpha)}\right.\right. \\
\left.\left.+\frac{x_{k+n-l}}{\tau_{k+n-l}(\alpha)}\right)+x_{k} y_{n} x_{l}\left(\frac{y_{k+n-l}}{\tau_{k+n-l}(\alpha)}+\frac{y_{n+l-k}}{\tau_{n+l-k}(\alpha)}+\frac{y_{k+l-n}}{\tau_{k+l-n}(\alpha)}\right)\right\} .
\end{array}
$$

Therefore, in this case, the terms that belong to $\mathcal{R}$ are $(k, l, n) \in \mathbb{N}^{3}$ in which $n=k$ and $n=l \neq k$. Thus, recalling that $\delta_{k, l}$ was introduced in (14), we have

$$
\hat{H}_{4}=\frac{b}{64} \sum_{(k, l) \in \mathbb{N}^{2}} \frac{4-\delta_{k, l}}{\tau_{k}^{2}(\alpha) \tau_{l}^{2}(\alpha)} x_{k} y_{k} x_{l} y_{l} .
$$

(3) If $\sigma=1$ and $\rho=0$ or $\sigma=1$ and $\rho=1$, proceeding in the same way as in case (2), we obtain that, in both cases,

$$
\hat{H}_{4}=\frac{b}{64} \sum_{(k, l) \in \mathbb{N}^{2}} \frac{4-\delta_{k, l}}{\tau_{k}^{2}(\alpha) \tau_{l}^{2}(\alpha)} x_{k} y_{k} x_{l} y_{l} .
$$

Thus, in the variables introduced in (14) and since $\tau_{k}(\alpha)=\left|\alpha-4 \pi^{2} k^{2}\right|^{1 / 2}$ for $k \in \Lambda(\alpha)$, the Hamiltonian $\bar{H}_{2}$ given in (19) becomes

$$
\bar{H}_{2}=\frac{1}{2} \sum_{k \in \Lambda(\alpha)}\left|\alpha-4 \pi^{2} k^{2}\right| \hat{I}_{k},
$$

$R$ given in (18) becomes $R\left(I_{k}, \theta_{k}\right), k=1, \ldots, k(\alpha)$, and, from (22), (23), taking the notation $\bar{H}_{4}=\Pi_{W_{c}}\left(\hat{H}_{4}\right)$, we have

$$
\bar{H}_{4}=-\frac{3 b}{64} \sum_{(k, l) \in \Lambda^{2}(\alpha)}\left(4-\delta_{k, l}\right) \hat{I}_{k} \hat{I}_{l} .
$$

This completes the proof of the lemma. 


\section{KAM theorem}

In this section we establish the existence of quasi-periodic space-symmetric solutions for the restriction to the center manifold of the Hamiltonian $H$ given in Section 2. Set $I=\left(\hat{I}_{1}, \ldots, \hat{I}_{k(\alpha)}\right), \theta=\left(\hat{\theta}_{1}, \ldots, \hat{\theta}_{k(\alpha)}\right)$ where $\left(\hat{I}_{k}, \hat{\theta}_{k}\right)$ were introduced in (14).

Proposition 3. For almost every $\alpha>0$, there exists a canonical change of variables $\mathcal{C}$ defined in a neighborhood of the origin such that

$$
H \circ \mathcal{C}=\bar{H}_{2}(I)+\bar{H}_{4}(I)+\cdots+\bar{H}_{2 N}(I)+\bar{R}(I, \theta)
$$

where $\bar{H}_{2 N}(I)$ are polynomials in the I-variables of degree $N$ vanishing at the origin and $\bar{R}(I, \theta)$ are divisible by $\sqrt{I^{N}}$.

The proof of the Proposition 3 is straightforward if one restricts the values of the parameter $\alpha$ to a full Lebesgue measure set.

From Proposition 3 and Lemma 2,

$$
\bar{H}_{2}(I)=\langle\tilde{\omega}, I\rangle, \quad \bar{H}_{4}(I)=\langle A I, I\rangle,
$$

with $\tilde{\omega}=\left(w_{1}^{2}(\alpha), \ldots, w_{k(\alpha)}^{2}\right)$ and

$$
A=\left(A_{k, j}\right)_{1 \leq k, j \leq k(\alpha)} \quad \text { in which } A_{k, j}=-\frac{3 b}{64}\left(4-\delta_{k, j}\right) .
$$

To establish the nondegeneracy conditions of KAM theory, we need to prove that $\operatorname{det}(A) \neq 0$. This is an immediate consequence of Corollary 6 in Appendix A, taking $d=-9 b / 64$ and $x=-12 b / 64$.

Once the Hamiltonian is transformed into the Birkhoff normal form up to order $2 \mathrm{~N}$ with the nondegeneracy property, we now need to prove the existence of invariant Cantor manifolds for Hamiltonians in such normal forms. This is deduced from the technical KAM theorem of Zehnder [13]. To state the quantitative version of the KAM theorem by Zehnder (see below), we recall that we say that the functions $F_{1}, F_{2}, \ldots$, $F_{n}$ are in involution if $\left\{F_{i}, F_{j}\right\}=0$ for $i \neq j$. Moreover, they are independent if the one-forms $d F_{1}, \ldots, d F_{n}$ are linearly independent over a full Lebesgue measure subset of the common definition domain of $F_{j}$ for $j=1, \ldots, n$. A Hamiltonian system with $n$ degrees of freedom having $n$ independent functions which are constant over the trajectories of the system and are in involution is called integrable.

Theorem 4. Let $F^{0}(\tilde{I})$ be a real analytic, integrable and nondegenerate (that is, $\operatorname{det}\left(\frac{\partial^{2} F^{0}}{\partial(\tilde{I})^{2}}(\tilde{I})\right) \neq 0$ ) Hamiltonian. Moreover, let the perturbed Hamiltonian $F(\tilde{I}, \tilde{\theta})=$ $F^{0}(\tilde{I})+F_{1}(\tilde{I}, \tilde{\theta})$ be of class $C^{l}, l \geq 4 \tau+10$, on

$$
\tilde{I} \in A_{m}=\left\{I_{j}>0: m \leq I_{j} \leq 2 m, 1 \leq j \leq N\right\}, \quad \tilde{\theta} \in \mathbb{T}^{N} .
$$


Then there exists $K$ depending on $\tau, \gamma$ such that, if $\left|F_{1}\right|_{C^{l}\left(A_{m} \times \mathbb{T}^{N}\right)} \leq K$, then all the invariant tori for the frequencies $w=\partial_{\tilde{I}} F^{0}(\tilde{I})$ satisfying

$$
|\langle w, k\rangle| \geq \gamma\|k\|^{-\tau} \quad \text { for all } k \in \mathbb{Z}^{N} \backslash\{0\}
$$

persist in the perturbed Hamiltonian $F(\tilde{I}, \tilde{\theta})$ in the following sense: there is a map $\phi: \mathbb{T}^{N} \rightarrow A_{m} \times \mathbb{T}^{N}$, where $\phi \in C^{m}\left(A_{m} \times \mathbb{T}^{N}\right)$ with $m=l-2 \tau-1$, and the flow on the invariant tori is the image by $\phi$ of the linear flow given by $\omega$.

We can finally show how Theorem 4 implies Theorem 1 .

Proof of Theorem 1. First of all, notice that from Proposition 3, the Hamiltonian

$$
\bar{H}_{2}(I)+\bar{H}_{4}(I)+\cdots+\bar{H}_{2 N}(I)
$$

is a real polynomial of degree $N$ in the $I$-variables and, thus, it is real analytic as a function of $I$. Furthermore it is integrable. From the hypothesis of Theorem 1, $w(\alpha)=\partial_{I} \bar{H}_{2}(I)$ satisfies (7), i.e.,

$$
|\langle w(\alpha), k\rangle| \geq \gamma\|k\|^{-\tau} \quad \text { for all } k \in \mathbb{Z}^{k(\alpha)} \backslash\{0\} .
$$

Thus, taking $m$ sufficiently small,

$$
w=\partial_{I}\left(\bar{H}_{2}(I)+\cdots+\bar{H}_{2 N}(I)\right)
$$

also satisfies (24). Moreover, as pointed out before, $\bar{H}_{2}(I)+\bar{H}_{4}(I)$ is nondegenerate. Therefore, taking $m$ sufficiently small, the Hamiltonian given in (25) is also nondegenerate.

By Proposition 3, we have that $\bar{R}(I, \theta)$ is divisible by $\sqrt{I^{N}}$, and, thus,

$$
\|\bar{R}\|_{C^{l}\left(A_{m} \times \mathbb{T}^{k(\alpha)}\right)} \leq C\left(\|\bar{R}\|_{C^{N}\left(A_{m} \times \mathbb{T}^{k(\alpha)}\right)}\right) m^{\frac{N}{2}-l} .
$$

Therefore, for a given $l \geq 4 \tau+10$, taking $N>2 l$ in Proposition 3, and also taking $m$ small enough, we can achieve

$$
\|\bar{R}\|_{C^{l}\left(A_{m} \times \mathbb{T}^{k(\alpha)}\right)} \leq K
$$

for any positive $K>0$. Therefore, taking $K$ sufficiently small, Theorem 4 implies that the Hamiltonian $H$ has $C^{l-2 \tau-2}$-invariant tori on which the flow is linear.

We want to mention that in [2], working with an analytic perturbed Hamiltonian $H(I, \theta)$, the authors prove that, in a neighborhood of given radius $m$, the measure of the complement of the KAM tori with frequencies satisfying a Diophantine condition with exponent $\gamma$ is exponentially small in $(1 / m)^{1 /(\gamma+1)}$. 


\section{Appendix A}

This appendix is devoted to the proof of a technical lemma that will be used to prove the nondegeneracy conditions necessary to apply KAM theory. Before stating it, let us introduce the set

$$
M_{n \times n}=\left\{A=\left(A_{i, j}\right)_{1 \leq i, j \leq n} \in \mathcal{M}_{n \times n}: A_{i, j}=\left\{\begin{array}{ll}
d, & \text { if } i=j, i \geq 1 \\
x, & \text { otherwise }
\end{array}\right\} .\right.
$$

Lemma 5. For any $n \geq 1$ and $A \in M_{n \times n}$,

$$
\operatorname{det}(A)=(d-x)^{n-1}(d+(n-1) x) .
$$

Corollary 6. For any $n \geq 1$ and $A \in M_{n \times n}$, we have $\operatorname{det}(A)=0$ if and only if $x=d$ or $x=-d /(n-1)$.

Proof of Lemma 5. In order to prove Lemma 5, we need to define an auxiliary matrix $B=\left(B_{i, j}\right)_{1 \leq i, j \leq n} \in \mathcal{M}_{n \times n}$ as

$$
B_{i, j}= \begin{cases}d, & \text { if } i=j, i \geq 2 \\ x, & \text { otherwise }\end{cases}
$$

Then we claim that

$$
\operatorname{det}(B)=x(d-x)^{n-1} .
$$

The proof of Lemma 5 will be done by proving also equation (26) simultaneously by induction over the dimension $n$.

For $n=1,2$, Lemma 5 and (26) hold trivially. Let us proceed by induction assuming that the case $n=m-1$ is proved and we shall prove it for $n=m$. We denote by $G^{(m-1)}$ and $G^{(m)}$ the determinant of $A$ for $n=m-1$ and $n=m$, respectively; and by $P^{(m-1)}$ and $P^{(m)}$ the determinant of $B$ at $n=m-1$ and $n=m$, respectively. Then we have

$$
G^{(m)}=d G^{(m-1)}-x(m-1) P^{(m-1)}, \quad P^{(m)}=x G^{(m-1)}-x(m-1) P^{(m-1)} .
$$

We start by proving the first identity. To this end we take the expansion (in the first row) on minors of the determinant, and we have,

$$
G^{(m)}=d G^{(m-1)}-x \sum_{k=2}^{m}(-1)^{k} \operatorname{det}\left(\Delta^{(k)}\right),
$$

where $\Delta^{(k)}=\left(\Delta_{i, j}^{(k)}\right)_{1 \leq i, j \leq m-1}$ with

$$
\Delta_{i, j}^{(k)}= \begin{cases}d, & j=i+1, i=1, \ldots, k-2 \text { for any } k \geq 3 \\ d, & j=i, i=k, \ldots, m-1, \\ x, & \text { otherwise. }\end{cases}
$$


We manipulate $\Delta^{(k)}$ as follows: we consider $\hat{\Delta}^{(k)}=\left(\hat{\Delta}_{\hat{i}, j}^{(k)}\right)_{1 \leq \hat{i}, j \leq m-1}$ with

$$
\begin{array}{ll}
\hat{\Delta}_{1, j}^{(k)}=\Delta_{k-1, j}^{(k)} & \text { for } j=1, \ldots, m-1, \\
\hat{\Delta}_{\hat{i}, j}^{(k)}=\Delta_{i-1, j}^{(k)} & \text { for } \hat{i}=2, \ldots, k-1, j=1, \ldots, m-1, \\
\hat{\Delta}_{\hat{i}, j}^{(k)}=\Delta_{i, j}^{(k)} & \text { for } \hat{i}=k, \ldots, m-1, j=1, \ldots, m-1 .
\end{array}
$$

Then

$$
\hat{\Delta}^{(k)}= \begin{cases}d, & j=\hat{i}, \hat{i} \geq 2, \\ x, & \text { otherwise, }\end{cases}
$$

which clearly coincides with $B=\left(B_{i, j}\right)_{1 \leq i, j \leq n-1}$. Moreover,

$$
\operatorname{det}\left(\Delta^{(k)}\right)=(-1)^{k-2} \operatorname{det}\left(\hat{\Delta}^{(k)}\right)=(-1)^{k-2} P^{(m-1)}
$$

and, thus,

$$
G^{(m)}=d G^{(m-1)}-x \sum_{k=2}^{m}(-1)^{2 k-2} P^{(m-1)}=d G^{(m-1)}-x(m-1) P^{(m-1)},
$$

which implies the desired relation. The second identity is obtained in the same way.

Therefore, from the inductive hypothesis and (26), $G^{(m)}$ is given by

$d(d-x)^{m-2}(d+(m-2) x)-x^{2}(m-1)(d-x)^{m-2}=(d-x)^{m-1}(d+(m-1) x)$

and

$$
P^{(m)}=x(d-x)^{m-2}(d+(m-2) x)-x^{2}(m-1)(d-x)^{m-2}=x(d-x)^{m-1} .
$$

\section{Appendix B}

For the convenience of the reader, we have included this appendix where we make precise the statement of the center manifold theorem and we present a sketch of the proof. We recall that $H_{0}^{m}([0,1])$ denotes the Sobolev space of functions on $[0,1]$ with periodic boundary conditions and zero mean.

Theorem 7. For each $r \geq 1$, there is a $C^{r}$ finite-dimensional manifold, $W_{c} \subset$ $H_{0}^{m-1}([0,1]) \times H_{0}^{m-2}([0,1])$, for $m \geq 2$, containing 0 , which is locally invariant under (1) in some neighborhood of the origin, and such that $T_{0} W_{c}$ is obtained from the spanning of the linear part of (1) with purely imaginary eigenvalues, i.e., the eigenvalues $\left\{\mu_{k}(\alpha)\right\}_{k<k(\alpha)}$ (see (4) and (5)). 
Proof. This theorem is a straightforward application of Theorem 2.1 in Mielke [9].

Theorem 8 ([9, Chapter 2]). Let X be a Hilbert space. We consider the system

$$
\frac{d x}{d t}=K x+f(t, \lambda, x)
$$

where $K: D(K) \rightarrow X$ is a closed linear operator, $f\left(t, \lambda_{0}, x\right)=\mathrm{O}\left(\|x\|^{2}\right)$, and $\lambda \in \mathbb{R}^{n}$ is a parameter. We assume the following hypotheses:

(1) The space $X$ splits into two closed subspaces $X_{1} \oplus X_{2}$ which are $K$-invariant:

$$
K_{j}=\left.K\right|_{X_{j}}: D(K) \cap X_{j} \rightarrow X_{j} \quad \text { for } j=1,2 .
$$

(2) The spectrum of the linear operator $K_{1}$ is contained in the imaginary axis, and $K_{1}$ is the generator of a strongly continuous group $\left(e^{K_{1} t}\right)_{t \in \mathbb{R}}$ satisfying

$$
\left\|e^{K_{1} t}\right\| \leq C(1+|t|)^{m} \quad \text { for some } C, m>0 .
$$

(3) The imaginary axis lies in the resolvent set of $K_{2}$ and for some $C>0$,

$$
\left\|\left(K_{2}-i \xi\right)^{-1}\right\| \leq \frac{C}{1+|\xi|}, \quad \xi \in \mathbb{R} .
$$

(4) There exist $k \in \mathbb{N}$ and a neighborhood $U \subset D(K)$ of 0 and a neighborhood $\lambda \subset \mathbb{R}^{n}$ of $\lambda_{0}$ such that $f=f(t, \lambda, x) \in C_{b, \text { unif }}^{k+1}(\mathbb{R} \times \Lambda \times U, X), f\left(t, \lambda_{0}, 0\right)=0$, and $D_{x} f\left(t, \lambda_{0}, 0\right)=0$ for all $t \in \mathbb{R}$.

Then there exist neighborhoods $\tilde{U}_{1} \subset U \cap X_{1}, \tilde{U}_{2} \subset U \cap X_{2}$ of 0 and $\tilde{\Lambda} \subset \Lambda$ of $\lambda_{0}$, and a function

$$
h=h\left(t, \lambda, x_{1}\right) \in C_{b, \text { unif }}^{k}\left(\mathbb{R} \times \tilde{\Lambda} \times \tilde{U}_{1}, \tilde{U}_{2}\right)
$$

with $h\left(t, \lambda_{0}, 0\right)=0$ and $D_{x_{1}} h\left(t, \lambda_{0}, 0\right)=0$ such that the graph of $h$,

$$
\left\{\left(t, x_{1}+h\left(t, \lambda, x_{1}\right)\right) \in \mathbb{R} \times X:\left(t, x_{1}\right) \in \mathbb{R} \times \tilde{U}_{1}\right\},
$$

is a locally invariant center manifold for (27).

We just need to verify the conditions in this result. The eigenvalues $\left\{\mu_{k}(\alpha)\right\}_{k \geq 1}$ of the linearized part

$$
K: H_{0}^{m}([0,1]) \times H_{0}^{m-1}([0,1]) \rightarrow H_{0}^{m-1}([0,1]) \times H_{0}^{m-2}([0,1]),
$$

with

$$
K(u, v)=\left(\begin{array}{c}
v \\
-\left(\partial^{2} / \partial x^{2}\right) u-\alpha u
\end{array}\right)
$$


are given in (4). Therefore, only finitely many lie on the imaginary axis, and there are no zero eigenvalues (since we have the zero mean condition). Furthermore, $K$ can be written as $K=K_{1}+K_{2}$ in which $K_{1}$ corresponds to the projection in the imaginary space and so $e^{t K_{1}}$ generates a strongly continuous group that satisfies $\left\|e^{K_{1} t}\right\| \leq C(1+|t|)^{m}$ for some $C, m>0$ and $K_{2}$ satisfies the resolvent estimate

$$
\left\|\left(K_{2}-i \xi\right)^{-1}\right\| \leq \frac{C}{1+\|\xi\|}, \quad \xi \in \mathbb{R},
$$

for some $C>0$. Furthermore, the nonlinearity $(0, g(u))$ is $C^{r+1}$ from

$$
H_{0}^{m}([0,1]) \times H_{0}^{m-1}([0,1]) \rightarrow H_{0}^{m-1}([0,1]) \times H_{0}^{m-2}([0,1]) .
$$

We have thus verified all the conditions which allow us to apply Theorem 8 . Our statement is simply a reformulation of this theorem.

Acknowledgements. The author wishes to thank Luis Barreira for his useful comments and remarks.

\section{References}

[1] À. Calsina, X. Mora and J. Solà-Morales, The dynamical approach to elliptic problems in cylindrical domains, and a study of their parabolic singular limit. J. Differential Equations 102 (1993), 244-304. Zbl 0803.35048 MR 1216730

[2] A. Delshams and P. Gutiérrez, Estimates on invariant tori near an elliptic equilibrium point of a Hamiltonian system. J. Differential Equations 131 (1996), 277-303. Zbl 0861.58016 MR 1419016

[3] M. Groves and J. Toland, On variational formulations for steady water waves. Arch. Rational Mech. Anal. 137 (1997), 203-226. Zbl 0888.76008 MR 1463795

[4] G. Iooss and K. Kirchgässner, Water waves for small surface tension: an approach via normal form. Proc. Roy. Soc. Edinburgh Sect. A 122 (1992), 267-299. Zbl 0767.76004 MR 1200201

[5] G. Iooss and A. Mielke, Bifurcating time-periodic solutions of Navier-Stokes equations in infinite cylinders. J. Nonlinear Sci. 1 (1991), 107-146. Zbl 0797.76010 MR 1102833

[6] K. Kirchgässner, Wave-solutions of reversible systems and applications. J. Differential Equations 45 (1982), 113-127. Zbl 0507.35033 MR 0662490

[7] S. Kuksin and J. Pöschel, Invariant Cantor manifolds of quasi-periodic oscillations for a nonlinear Schrödinger equation. Ann. of Math. (2) 143 (1996), 149-179. Zbl 0847.35130 MR 1370761

[8] A. Mielke, Reduction of quasilinear elliptic equations in cylindrical domains with applications. Math. Methods Appl. Sci. 10 (1988), 51-66. Zbl 0647.35034 MR 0929221

[9] A. Mielke, Hamiltonian and Lagrangian Flows on Center Manifolds. Lecture Notes in Math. 1489, Springer-Verlag, Berlin 1991. Zbl 0747.58001 MR 1165943 
[10] A. Mielke, Essential manifolds for an elliptic problem in an infinite strip. J. Differential Equations 110 (1994), 322-355. Zbl 0814.35028 MR 1278374

[11] C. Siegel and J. Moser Lectures on Celestial Mechanics. Grundlehren Math. Wiss. 187, Springer-Verlag, New York, Heidelberg 1971. Zbl 0312.70017 MR 0502448

[12] C. Wayne, An introduction to KAM theory. In Dynamical Systems and Probabilistic Methods in Partial Differential Equations (Berkeley, USA, 1994), Lectures in Appl. Math. 31, Amer. Math. Soc., Providence, RI, 1996, 3-29. Zbl 0844.58037 MR 1363023

[13] E. Zehnder, Generalized implicit function theorems with applications to some small divisor problems. II. Comm. Pure Appl. Math. 29 (1976), 49-111. Zbl 0334.58009 MR 0426055

Received July 3, 2004

Claudia Valls, Departamento de Matemática, Instituto Superior Técnico, 1049-001 Lisboa, Portugal

E-mail: cvalls@math.ist.utl.pt 